\title{
Markers of Neuroinflammation and Apoptosis in the Temporal Lobe of Patients with Drug-Resistant Epilepsy
}

\author{
A. V. Litovchenko ${ }^{a, b, *}$, Yu. M. Zabrodskaya ${ }^{b, c}$, D. A. Sitovskaya ${ }^{c}$, L. K. Khuzhakhmetova ${ }^{d}$, \\ V. G. Nezdorovina ${ }^{c}$, and E. D. Bazhanova ${ }^{a, b, e}$ \\ ${ }^{a}$ Sechenov Institute of Evolutionary Physiology and Biochemistry, Russian Academy of Sciences, \\ St. Petersburg, Russia \\ ${ }^{b}$ Golikov Research Clinical Center of Toxicology, Federal Medical Biological Agency, \\ St. Petersburg, Russia \\ ${ }^{c}$ Polenov Neurosurgical Institute, Almazov National Medical Research Center, St. Petersburg, \\ Russia \\ ${ }^{d}$ Astrakhan State University, Astrakhan, Russia \\ e Astrakhan State Medical University, Astrakhan, Russia \\ *e-mail: anastasiya_litovchenkos@list.ru
}

Received March 29, 2021

Revised June 3, 2021

Accepted June 4, 2021

\begin{abstract}
Current antiepileptic strategies aim to normalize the interaction of the excitatory and inhibitory systems, which is ineffective in treating patients with drug-resistant epilepsy. Neuroinflammatory processes in the epileptic focus and its perifocal area can trigger apoptosis and also contribute to the development of drug resistance. The level of pro- and anti-apoptotic proteins (p-NF-kB, TNF- $\alpha, \mathrm{p} 53$, FAS, caspase-3, caspase-9) was analyzed in intraoperative biopsies of the temporal lobe gray and white matter in the brain of patients with drug-resistant epilepsy. An increased level of pro-apoptotic proteins was revealed in the cortex and perifocal area's white matter against the background of an imbalance of protective anti-apoptotic proteins. It appears that the activation of the extrinsic pathway of apoptosis occurs in the perifocal area, while in the epileptic focus, there are proteins responsible for the activation of the anti-apoptotic survival pathways. Active neuroinflammation in the epileptic focus and perifocal area of the temporal lobe may contribute to the development of the resistance to antiepileptic drugs and the progression of neurodegeneration in such patients.
\end{abstract}

DOI: $10.1134 / \mathrm{S} 0022093021050069$

Keywords: drug resistance, temporal lobe epilepsy, apoptosis, neuroinflammation, cytokines, immunoblotting

\section{INTRODUCTION}

Every year, around 2 million new cases of epi- lepsy are diagnosed in the world. At the same time, the International League Against Epilepsy (ILAE) established that in young patients with 
epilepsy the mortality rate is 4 times that in the same age group [1]. The incidence of drug-resistant epilepsy (DRE) that requires surgical treatment accounts for $30 \%$ of the total number of patients. According to clinical studies, the proportion of drug-resistant forms has been remaining invariable over the past 30 years, despite the emergence of new antiepileptic drugs with basically different mechanisms of action [2]. Drugresistant forms of epilepsy are characterized by rapid progression and significantly reduce both the patients' quality of life and their work capacity [3]. Surgical treatment, which is indicated with focal DRE, in addition to being traumatic, leads to positive outcomes only in $60 \%$ of cases, of which the number of seizures decreases in $20 \%$ of them, while in $20 \%$ the disease relapses [4]. These data confirm the urgent need to seek for new pathogenetic mechanisms of epilepsy.

Epileptogenesis is a dynamic process that gradually alters the excitability of neurons, establishes critical relationships, and requires complex structural changes before the first spontaneous seizure occurs [5]. These changes may concern neurodegeneration, neurogenesis, gliosis, axonal injury, dendritic plasticity, blood-brain barrier (BBB) lesions, inflammatory cell recruitment into brain tissue, reorganization of the extracellular matrix and molecular functioning of individual neurons [6].

The molecular mechanisms leading to a change in the pharmacosensitivity of neurons to modern drugs are still unknown. Presumably, the cellular mechanism of drug resistance in chronic epilepsy may consist in a reduction in the number of voltage-gated channels, as revealed in both experimental models of epilepsy and clinical studies [7]. Neuroinflammation, which regularly occurs in patients with other neurodegenerative diseases (Alzheimer's, Parkinson's, Huntington's, Pick's disease, etc.), may represent the key mechanism of damaging voltage-gated channels and neuronal death in the context of the development of drugresistant forms of epilepsy [8]. In preclinical trials, the use of non-steroidal anti-inflammatory drugs (NSAIDs) as agents that reduce convulsive activity did not reduce the proportion of rats who developed epilepsy, however, reduced the frequency and duration of seizures, the level of microglial activation and leukocyte infiltration, and also suppressed oxidative stress and the release of cytokines, contributed to the reduction of neurodegeneration [9].

Neuroinflammation and apoptosis of nerve cells may play a significant role in the pathogenesis of epilepsy. Recent data confirm the active process of death of brain neurons in the temporallobe form of this disease [10]. For example, in rodents, an increased expression of the proinflammatory cytokines interleukin IL-1 $\beta$ and tumor necrosis factor (TNF) was shown in the brain, leading to neuroinflammation in an animal model of focal cerebral ischemia [11]. In the brain, resident cellular components contain several so-called death receptors, such as TNFR1, FAS, and DR4, which, when interacting with TNF- $\alpha$ and FAS-L, activate the extrinsic receptor pathway of apoptosis [12]. The receptor mechanism of apoptosis in epilepsy was previously confirmed indirectly by administering TNF- $\alpha-$ neutralizing antibodies having an in vitro neuroprotective effect [13].

Some studies have reported the association of the nuclear factor NF-kB, a master regulator of the inflammatory response, with epilepsy in animals [14]. NF-kB consists of two subunits, and its most common form is the p65/p50 heterodimer, which typically exists in the cytoplasm in a form inhibited by the IkB protein. Stimulation of cell surface receptors by such factors as oxidative stress, cytokines or pathogens activates the IkB complex. Proteasome degradation of IkB proteins leads to the release and translocation of NF-kB dimers into the nucleus, where it promotes the transcription of target genes to counteract oxidative stress and cell damage [15]. Recent studies have confirmed NF-kB activation in most cell types, including neurons, astrocytes, microglia, and oligodendrocytes [16]. In vitro, NF-kB activation was found in cultured neurons under the influence of glutamate and $\beta$-amyloid. In vivo, a similar picture was observed under conditions of experimental autoimmune encephalomyelitis, cerebral ischemia, and Alzheimer's disease [17]. Thus, NF-kB plays an important role as a signaling pathway in the central nervous system, activating survival cascades in response to neuronal damage. 
In contrast to the activation of survival pathways, nerve cells can undergo apoptosis in response to external stimulation. Apoptosis can be activated via the extrinsic (receptor) and mitochondrial pathways. While the mitochondrial pathway of apoptosis is often associated with oxidative stress at the cellular level, then the extrinsic pathway is activated due to a damage or cellular immune response [18]. Microglia can function as a substrate of cellular immunity in the brain, and its activation leads to the secretion of pro-inflammatory factors in nervous tissue and causes neuroinflammation and apoptosis [19].

The present work aimed to identify the molecular mechanisms underlying the pathogenesis of human DRE. Our objectives included an elucidation of the possible role of TNF- and NF-kBdependent signaling pathways in the expression of apoptosis-associated molecules (p53, caspase-9 and -3 ) in nervous tissue of the temporal lobe in patients with DRE.

\section{MATERIALS AND METHODS}

All patients included in this study were observed in the clinic of the Polenov Neurosurgical Institute, which is a branch of the Almazov National Medical Research Center (St. Petersburg), in 2013-2020. During this period, 24 patients were examined and surgically treated (mean age $27.75 \pm 2.46$ years; 9 women and 15 men) with an established diagnosis according to International Statistical Classification of Diseases and Related Health Problems classification G40.2 "Temporal-lobe focal epilepsy of structural etiology with frequent polymorphic seizures, pharmacoresistant course". The patients were added to our study sequentially; the inclusion criteria were the epileptic activity predominantly in the temporal lobe of the brain, as confirmed by invasive EEG monitoring, and the sufficiency of the biopsy material obtained during surgery (e.g., en bloc resection of the temporal lobe) with informed consent from each patient to sample, transport, store and examine biopsy specimens. In the case of insufficient biological material (e.g., due to stereotaxic intervention or pronounced fragmentation of biopsy specimens), in the presence of neoplasms or brain infection, or with autoimmune diseases or immunodeficiency states in the anamnesis, the patients were excluded from the study. In these patients, anteromedial temporal resection was carried out under electrophysiological control in accordance with their diagnosis and EEG examination data. The epileptic focus area was determined using the MRI Epilepsy program, PET-CT, and invasive EEG monitoring. When performing invasive EEG monitoring, the gyrus involvement in the epileptic process was determined. During surgical intervention, under the control of electrocorticography, the area of epileptiform activity was specified and recorded in the surgical intervention protocol, with en bloc resection of the anterior portion of the temporal lobe being performed thereafter. In the Morbid Anatomy Department, the removed fragment of the brain matter was anatomically oriented, measured and photographed. According to the surgical intervention protocol, the epileptic focal area was determined to make a vertical incision therethrough, forming a lamina $0.2 \mathrm{~cm}$ thick. For further examination, fragments of the gyral cortex and underlying white matter were sampled directly from the epileptic focus and perifocal area, then labeled and frozen.

The comparison group comprised 6 patients (mean age $28.75 \pm 3.73$ years; 2 women and 4 men), comparable with the study group in their sex and age, operated for craniocerebral trauma, and included into this group on condition that they had no epileptic syndrome in the anamnesis, nervous system pathologies (tumors, brain infarction, stroke, infections), autoimmune diseases or immunodeficiency states. Patients of this group were hospitalized for reconstructive interventions (plasty of cranial vault defects, surgical excision of adhesions in the gliomesenchymal scar area) in a distant post-traumatic period (0.5-1 year). Patients with acute craniocerebral trauma were excluded from the study. All specimens of the cortex and white matter were stored at $-80^{\circ} \mathrm{C}$.

A fragment weighing $0.03-0.05 \mathrm{~g}$ was selected from the surgical biopsies as quickly as possible at low temperatures. Then, a lysate buffer was added to the fragment in a ratio of $1: 10$, mixed ex tempore with inhibitors of proteases (Protease Inhibitor Cocktail, Sigma-Aldrich) and phosphatases (PhosSTOP, Sigma-Aldrich), and finally homog- 
enized. The resulting homogenate was kept cold for $30 \mathrm{~min}$ and then centrifuged for $15 \mathrm{~min}$ at $4^{\circ} \mathrm{C}$ at a relative centrifugal acceleration of $12000 \mathrm{~g}$. At the end of centrifugation, the resulting supernatant was aspirated, mixed with a Laemmli buffer solution, and incubated for $5 \mathrm{~min}$ at $95^{\circ} \mathrm{C}$.

Levels of the following proteins were studied by immunoblotting: NF-kB p65 (phospho S536) (1: 500), FAS (1: 1000), p53 (1: 250), TNF- $\alpha$ (1: 1000), STAT1 (1 : 1000), caspase-9 (1: 1000) (Abcam, USA), caspase-3 (1: 1000), tubulin (1 : 1000) (Cell Signaling Technology, USA). Incubation with primary antibodies was carried out at $4^{\circ} \mathrm{C}$ for $8-12 \mathrm{~h}$ with constant stirring. To conduct the chemiluminescence reaction after incubation with secondary antibodies, a luminol-based enhanced chemiluminescence (ECL) horseradish peroxidase (HRP) substrate (SuperSignal West Dura Extended Duration Substrate; Thermo Scientific, USA) was used. The reaction was assessed using the ChemiDoc gel documentation imaging system (BioRad) with subsequent densitometry of the resulting blots using the Image J software. Statistical data processing was carried out using the GraphPad Prism 8.0.1 software package. For quantitative variables, the significance of differences between unrelated groups was assessed using the Kruskal-Wallis test. The differences were considered statistically significant at $p<0.05$.

For immunohistochemical (IHC) analysis of the expression of apoptosis proteins, the temporal lobe biopsy, obtained by temporal en bloc resection, was fixed in $10 \%$ buffered neutral formalin (pH 7.4), embedded in paraffin according to a standard technique, and cut into frontal sections 6-7 $\mu \mathrm{m}$ thick. As a comparison group, we used the autopsy material from 5 patients $(3$ men, 2 women), aged 40 to 67 years (mean age $54 \pm$ 7.25), who died of trauma and had no neurological problems in their medical history, including epilepsy. The post-mortem delay before autopsy ranged from 7 to $12 \mathrm{~h}$ (on average $8.65 \pm 2.2$ ).

Protein antigenic epitopes were unmasked in citrate buffer at $95 \mathrm{C}$ for $20 \mathrm{~min}$. The IHC reaction was performed using rabbit polyclonal antibodies to caspase-3 (1:250) (Cell Signaling Technology, USA). Sections were incubated first with primary antibodies for $12 \mathrm{~h}$ at room temperature, then with secondary antibodies for $1 \mathrm{~h}$ at the same temperature, and finally in Streptavidin-Peroxidase Polymer, Ultrasensitive (Sigma-Aldrich, USA). The DAB Liquid Substrate System (SigmaAldrich, USA) was used for visualization. After the IHC reaction, the sections were counterstained with Gill's hematoxylin and embedded in a synthetic embedding medium Bio Mount HM (BIO-OPTICA Milano, Italy). The reaction outcomes were evaluated by counting the number of stained nuclei $(\%)$ per 100 cells in 10 visual fields in each case.

This work was implemented in accordance with ethical standards based on the principles of voluntariness and confidentiality. An informed consent was obtained from each patient. The experimental protocol was authorized by the Ethics Committee of the A.L. Polenov Neurosurgical Institute (Extract from the minutes No. 0305-2016 dated May 16, 2016). The study was carried out as part of the provision of high-tech medical care.

\section{RESULTS AND DISCUSSION}

Our results demonstrated the presence of neuroinflammation and apoptosis in brain tissues. Rana and Musto [20] reported that in patients hospitalized with convulsive syndrome, the level of the pro-inflammatory factor TNF- $\alpha$ in the cerebrospinal fluid and blood was increased, while in the rat hippocampus, TNF- $\alpha$ expression also rose after seizures. However, no comparison has been made so far between the level of this protein directly in the cortex and white matter of epileptic focal and perifocal areas in the human temporal lobe and the TNF- $\alpha$ level in the cortex and white matter of healthy living people. It is well known that the pro-inflammatory cytokine TNF- $\alpha$ is released in the brain from activated microglia and astrocytes [21]. In our specimens, an increased TNF- $\alpha$ level was revealed in epileptic focal and perifocal areas of the temporal lobe cortex and white matter (Fig. 1). Likewise, an increased expression of the FAS receptor was detected in the epileptic focus of the temporal lobe gray and white matter as compared to the control group. In the perifocal area, FAS expression was increased in the cortex only, while in the white matter, an upward trend was observed. In the studies by 

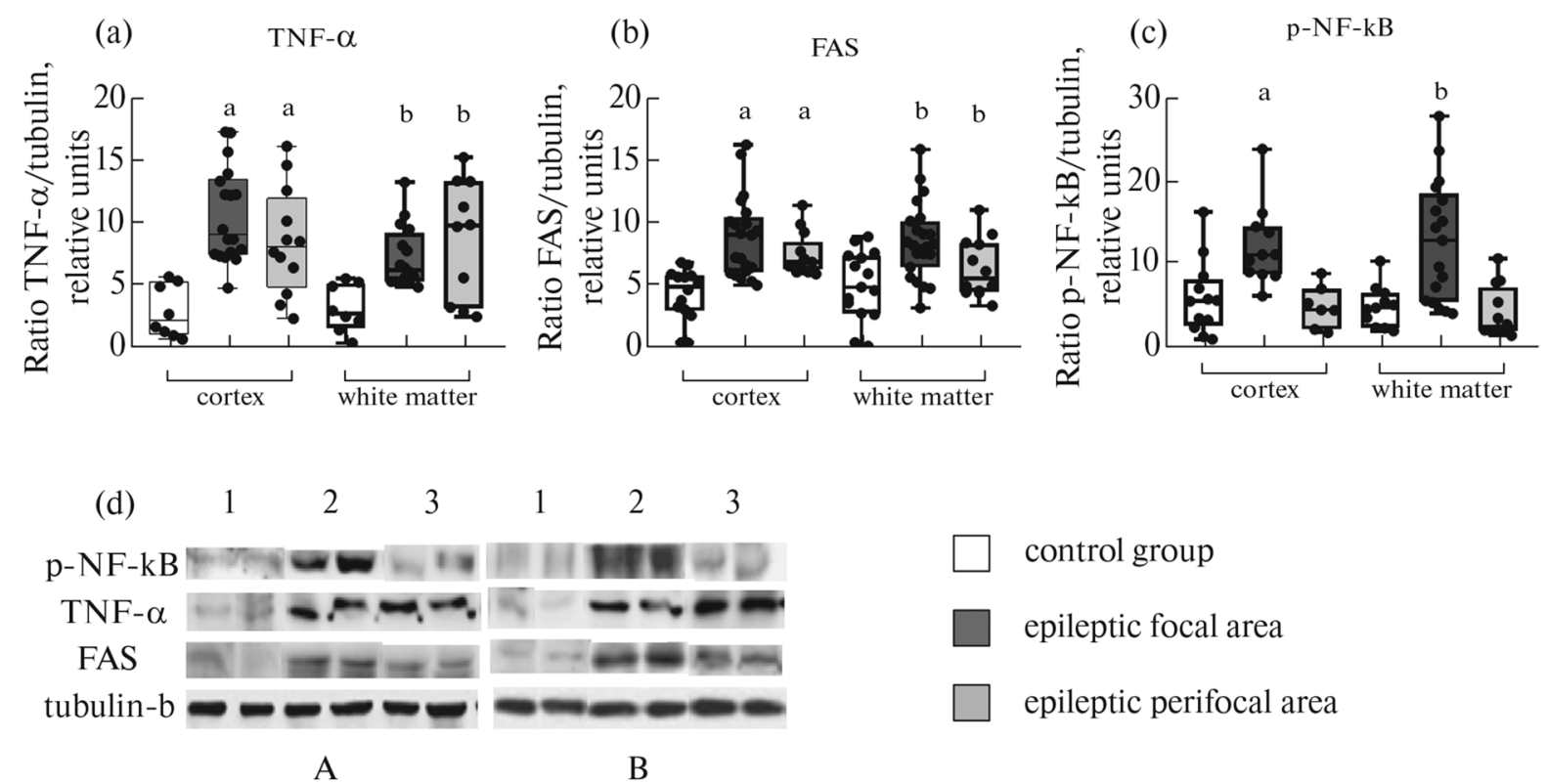

Fig. 1. Relative content of TNF- $\alpha$ (a), FAS (b), and p-NF-kB (c) in the cortex and white matter of the temporal lobe. Lysates of human temporal lobe cortex and white matter biopsies examined by immunoblotting in $10 \%$ polyacrylamide gel (d). (a) Differences between the cortex vs. control group, $p<0.05$; (b) differences between the white matter vs. control group, $p<$ 0.05 ; (c) differences between the perifocal cortical area vs. the epileptic focus, $p<0.05$; (d) differences between the perifocal area white matter vs. the epileptic focus, $p<0.05$.

other authors, in patients with temporal lobe epilepsy, there was revealed an increased level of the TNF- $\alpha$ receptor (TNFR1) and FAS-associated protein with death domain (FADD) [22]. A high TNF- $\alpha$ level that we found in biopsies of the temporal lobe cortex and white matter against the background of increased expression of the FAS-L receptor may indicate the activation of immune cells in the brain of patients with DRE, as well as the extrinsic pathway of apoptosis directly in the cortex and white matter of the epileptic focus. In the perifocal area, these processes may also proceed, but less intensively. TNF- $\alpha$ binds to the matching receptor (TNFR1) and activates the TNFR-associated domain of the TRADD protein, while FAS-L interacts with the FAS-associated domain of the FADD protein [23]. The TNF- $\alpha$ activates adapter TRADD and thus enables competitive parallel pathways to suppress cell death caused by phosphorylation of the inhibitor protein to which NF-kB is bound. This phenomenon may explain the data on differential effects of TNF- $\alpha$ on apoptosis. In this case, the FAS/FAS-L system does not initiate any parallel pathways other than apoptotic [24].
The transcription factor $\mathrm{NF}-\mathrm{kB}$ regulates the expression of anti-apoptotic proteins [14]. TRADD-mediated degradation of the NF-kBIkB complex allows the NF-kB p65 isoform to translocate into the nucleus, where it regulates the transcription of a wide range of genes responsible for the synthesis of proteins, such as Bcl-2, IL-6, IL-8, and VEGF, which are involved in cell survival [25]. The p65 structure includes a C-terminal domain of 30 amino acids, which constitutes the most important site for NF-kB transactivation. Phosphorylation of the S536 site leads to an acceleration of the transcription of survival genes, or activates the NF-kB pathway outside IkB regulation [26].

In our specimens, in the epileptic focus of the cortex and white matter, the content of the S536phosphorylated NF-kB form was observed to be increased, while in the perifocal area, the content of this protein remained at the same level as in patients without epilepsy. A significant content of the phosphorylated NF-kB form directly in the epileptic focus may indicate the TNF- $\alpha$-induced activation of transcription of pro-inflammatory and anti-apoptotic genes, which leads largely not 

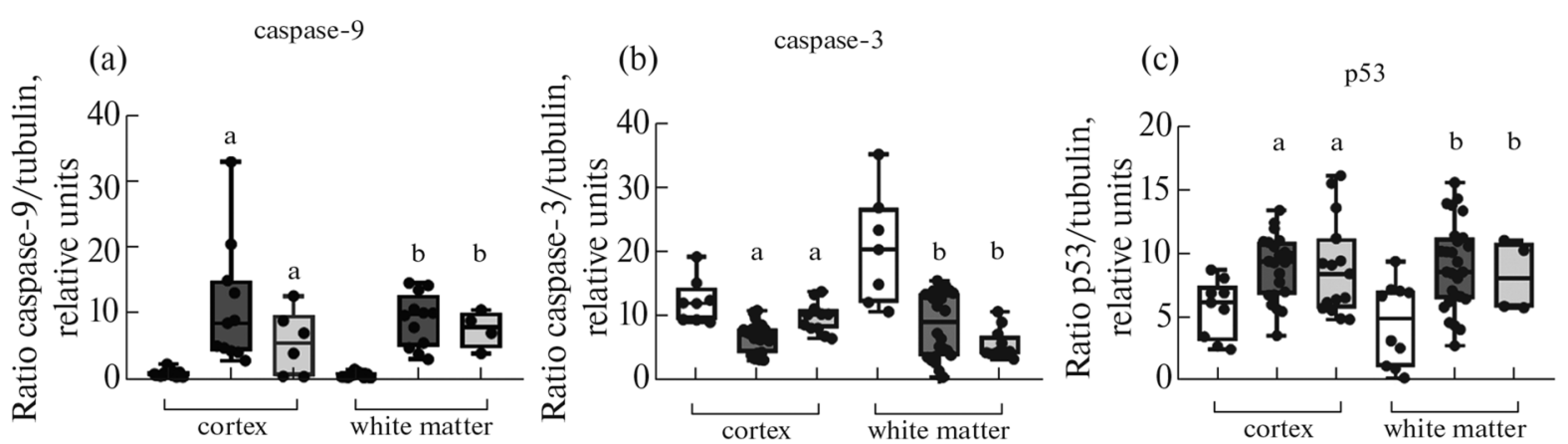



A

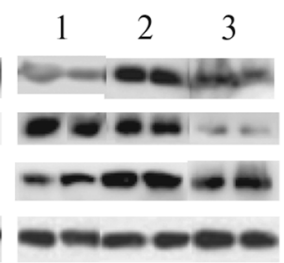

B

Fig. 2. Relative content of caspase-3 (a), caspase-9 (b), and p-53 (c) in the cortex and white matter of the temporal lobe. Lysates of human temporal lobe cortex and white matter biopsies examined by immunoblotting in $10 \%$ polyacrylamide gel (d). (a) Differences between the cortex vs. control group, $p<0.05$; (b) differences between the white matter vs. control group, $p<0.05$; (c) differences between the cortical perifocal area vs. epileptic focus, $p<0.05$; (d) differences between the white matter perifocal area vs. epileptic focus, $p<0.05$.

to cell death, but to their survival. However, a high TNF- $\alpha$ level that we revealed in in this study may indicate the presence of neuroinflammation in the case of DRE. In the epileptic perifocal area, at the same relative TNF- $\alpha$ concentration, triggering of the parallel survival pathway does not appear to occur, since no difference in the level of the phosphorylated NF-kB form between the control and DRE groups was found, from which we can conclude that in the perifocal area, against the background of neuroinflammation, the development of apoptosis is not restrained by protective pathways.

The revealed increased expression of the FAS receptor in the epileptic focus of the cortex and white matter, as well as in the perifocal area of the cortex, can be due to transcription of the p53 gene. The $\mathrm{p} 53$ protein that triggers the transcription of this gene is in a latent state in the cytoplasm and can be activated not only in response to DNA damage, but also due to the effect of oxidative stress, pathogenic or cytotoxic agents [27]. Apoptosis initiation through p53 activation occurs in several ways, one of which induces FAS mRNA and translocation of the synthesized FAS to the cell surface [28]. In addition, p53 acts directly on the outer mitochondrial membrane, and once inside the organelle, it interacts with $\mathrm{Bcl}-2$ or Bak, leading to the release of cytochrome $\mathrm{C}$ and activation of caspase-3 [18]. In the present study, an elevated p53 level was found in epileptic focal and perifocal areas of the temporal cortex and white matter (Fig. 2), which may be one of the reasons for the increase in FAS on the cell membrane, stimulating thereby the development of apoptosis under the effect of extrinsic factors. In this case, the effect of p53 on mitochondria acts as an additional factor in the activation of caspases and cell death.

The interaction of p53 with the membrane of a damaged mitochondria and the resulting cytochrome $\mathrm{C}$ release lead to the formation of a specific complex, apoptosome, which comprises the APAF-1 protein (apoptotic protease-activating factor 1), cytochrome $\mathrm{C}$ itself, and procaspase-9. The fusion of these proteins into the apoptosome leads to the activation of caspase-9. Caspase-9, in turn, activates effector caspases, including caspase-3, which release a bound DNase that destroys chromatin [23]. 


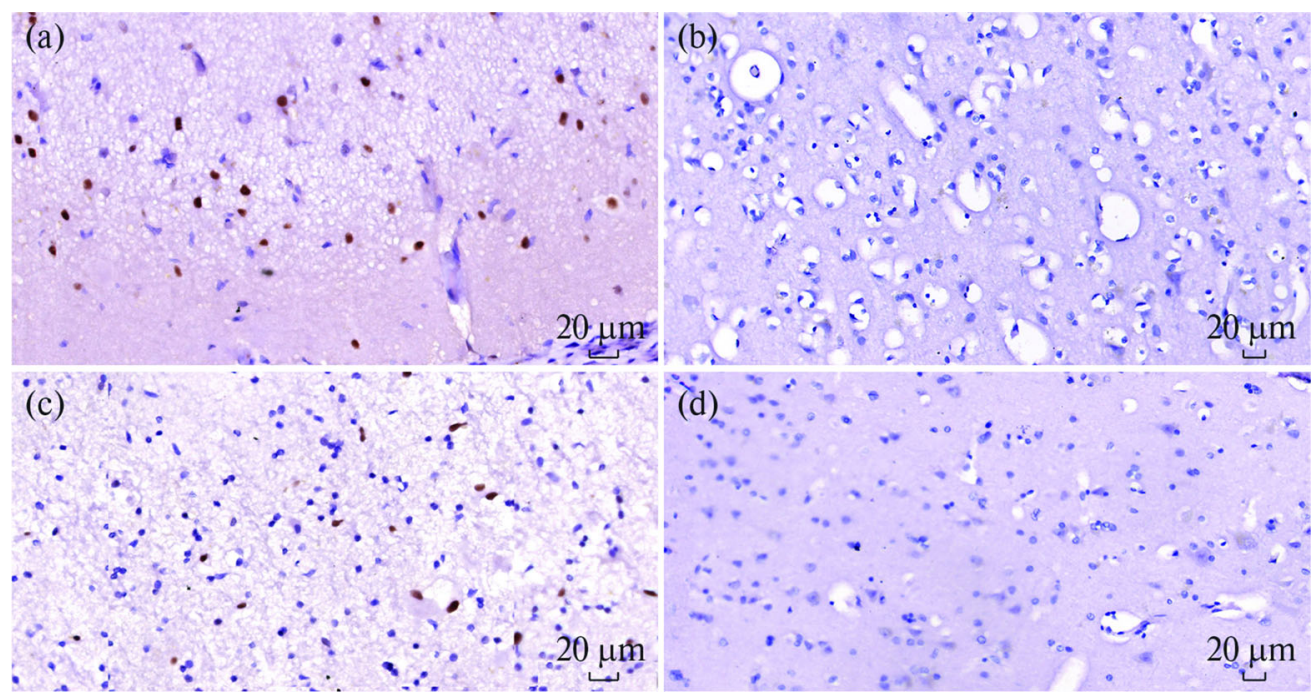

Fig. 3. Immunohistochemical reaction for caspase-3 in the temporal lobe cortical epileptic focus of a patient with (a) and without (b) drug-resistant epilepsy, as well as in the white matter perifocal area of a patient with drug-resistant epilepsy (c) vs. control white matter (d). DAB visualization (brown staining), Gill's hematoxylin counterstaining; magnification $\times 400$.

In the gray matter specimens from the epileptic focus, a high content of the initiator caspase-9 was revealed compared to the control group, while the caspase- 3 level in the same patients was reduced (Fig. 2). In the perifocal area of the gray matter, caspase- 9 and caspase- 3 levels obeyed the same tendency, but in a less pronounced manner. In epileptic focal and perifocal areas of the white matter, the same situation was observed. A reduced level of the effector caspase- 3 combined with a simultaneous elevation of caspase- 9 may be caused by the appearance of a cleaved caspase- 3 form (c-caspase-3), indirectly indicating apoptosis activation via the mitochondrial pathway [10]. In our previous study using the same methods, we revealed an increased caspase- 8 level in the epileptic focal and perifocal cortical areas in patients with DRE [29], indicative of the activation of apoptotic signaling pathways. In an experiment on rats, it was shown that caspase- 8 inhibitors reduce the protease activity of caspase- 3 and caspase-9, while caspase- 9 inhibitors, as applied in temporal lobe epilepsy, affect effector caspase3 activity just insignificantly [22]. Thus, it can be concluded that in temporal lobe epilepsy, the extrinsic receptor pathway of apoptosis is dominant. The activation of the mitochondrial pathway in temporal lobe epilepsy is also evidenced by other experiments, which showed the presence of the pro-apoptotic protein Bax in the outer mem- brane of mitochondria after the onset of a seizure $[10,20]$. In turn, mitochondrial dysfunction is one of the key pathogenic mechanisms of neurodegeneration [22].

Based on our data, it can be hypothesized that apoptosis proceeds more intensively in the perifocal area of excitation, since no increased level of protective NF-kB cascade proteins was detected in the perifocal area, and hence it is assumed that the survival pathways were not activated sufficiently. In this case, the perifocal area was also liable to the effect of inflammatory factors, likewise the focal areas. The involvement of perifocal areas in inflammatory processes, as well as the lack of protective mechanisms, may be among the factors that promote the development of DRE.

To verify the cell specificity of the expression of pro-apoptotic proteins, an IHC study of biopsies obtained by en bloc resection of the temporal lobe was performed. During IHC staining, in all patients with DRE, a positive nuclear expression of caspase-3 was observed not only in neurons, but also in glial cells of the cortex and white matter. In the cortex, gliocytes showed a high intensity of nuclear staining, their distribution was diffuse in all layers of the cortex, but mainly in the molecular layer (Fig. 3a). The mean relative count of caspase-3-immunopositive glial cells in the cortex of patients with DRE was $23.65 \pm$ $0.82 \%$. In the white matter, the nuclear expres- 
sion of caspase-3 was moderately pronounced, with the mean relative count of immunostained nuclei in glial cells of the white matter being somewhat lower than in the cortex, amounting to $17.45 \pm 0.54 \%$ (Fig. 3c). In the control group, caspase-3 expression in the white matter was not detected (Fig. 3d), while in the cortex, solitary immunostained (mainly in the cytoplasm) cells were observed, with no nuclear expression of caspase-3 being found (Fig. 3b). Thus, the detected expression of caspase- 3 in cortical and white matter gliocytes in the temporal lobe epileptic focus may be an indicator of the involvement of glia in apoptotic processes.

Previous studies of patients with DRE, as well as the experiments on animals, indicate an increase in the expression of pro-apoptotic proteins in the hippocampus [18, 20]. In the present work, we divided the study area into an epileptic focus and a perifocal area. The results showed that increased expression of protective p-NF-kB, caspase-9, pro-inflammatory $\mathrm{TNF}-\alpha$, and FAS receptor under the influence of p53 is observed in the epileptic focus in patients with temporal lobe DRE. It can be concluded that the neuroinflammation process occurs in the epileptic focus, leading to apoptosis via the extrinsic (receptor) and mitochondrial pathways, among which the extrinsic one predominates. In the perifocal area, the expression of pro-apoptotic proteins, although less intense, was also observed, while no activation of the NF-kB pathway's protective mechanisms was detected. Thus, active neuroinflammation in epileptic focal and perifocal areas of the temporal lobe, as well as the imbalance of the anti-apoptotic system in the perifocal area, promote further degeneration in the focus of hyperexcitability and the progression of epileptic encephalopathy, being therefore a possible cause for the development of therapeutic (drug) resistance.

\section{AUTHORS' CONTRIBUTION}

The idea and design: E.D.B. and Yu.M.Z.; patients management, surgery, sampling of the biological material: V.G.N. and D.A.S.; data collection and processing: A.V.L. and L.K.Kh.; writing and editing a manuscript: A.V.L., E.D.B., Yu.M.Z.

\section{FUNDING}

This work was supported by the Russian Foundation for Basic Research grant No. 20-015-00127, as well as by state budget funding on the topic No. 05600109-21-01(19). The part of the work performed at the Sechenov Institute of Evolutionary Physiology and Biochemistry $(\mathrm{IEPhB})$ was state budget-funded within the state assignment to IEPhB. Experiments were carried out on the equipment of the IEPhB Center for Collective Use.

\section{CONFLICT OF INTEREST}

The authors declare that they have no conflict of interest related to the publication of this article.

\section{OPEN ACCESS}

This article is licensed under a Creative Commons Attribution 4.0 International License, which permits use, sharing, adaptation, distribution and reproduction in any medium or format, as long as you give appropriate credit to the original author(s) and the source, provide a link to the Creative Commons license, and indicate if changes were made. The images or other third party material in this article are included in the article's Creative Commons license, unless indicated otherwise in a credit line to the material. If material is not included in the article's Creative Commons license and your intended use is not permitted by statutory regulation or exceeds the permitted use, you will need to obtain permission directly from the copyright holder. To view a copy of this license, visit http://creativecommons.org/ licenses/by/4.0/.

\section{REFERENCES}

1. Gromov SA, Sivakova NA (2019) Resolved epilepsy: an updated insight. Epilepsy and paroxysmal conditions 11:21-26 (In Russ). https:// doi.org/10.17749/2077-8333.2019.11.1.21-26

2. Chen Z, Brodie MJ, Liew D, Kwan P (2018) Treatment outcomes in patients with newly diagnosed epilepsy treated with established and new antiepileptic drugs: A 30-year longitudinal cohort study. JAMA Neurology 75:279-286. https:// doi.org/10.1001/jamaneurol.2017.3949

JOURNAL OF EVOLUTIONARY BIOCHEMISTRY AND PHYSIOLOGY Vol. 57 No. 52021 
3. Lotfinia M, Maloumeh EN, Asaadi S, Omidbeigi M, Sharifi G, Asadi B (2019) Healthrelated quality of life after epilepsy surgery: A prospective, controlled follow-up on the Iranian population. Scientific reports 9:7875. https://doi.org/ 10.1038/s41598-019-44442-6

4. Hader WJ, Tellez-Zenteno J, Metcalfe A, Hernandez-Ronquillo L, Wiebe S, Kwon C-S, Jette N (2013) Complications of epilepsy surgery: A systematic review of focal surgical resections and invasive EEG monitoring. Epilepsia 54:840847. https://doi.org/10.1111/epi.12161

5. Younus I, Reddy DS (2017) Epigenetic interventions for epileptogenesis: A new frontier for curing epilepsy. Pharmacology \& Therapeutics 177:108122. j.pharmthera.2017.03.002

https://doi.org/10.1016/

6. Sharma S, Puttachary S, Thippeswamy T (2019) Glial source of nitric oxide in epileptogenesis: A target for disease modification in epilepsy. Journal of neuroscience research 97:1363-1377. https:// doi.org/10.1002/jnr.24205

7. Zavala-Tecuapetla C, Cuellar-Herrera M, LunaMunguia H (2020) Insights into Potential Targets for Therapeutic Intervention in Epilepsy. International journal of molecular sciences 21:8573. https://doi.org/10.3390/ijms21228573

8. Luscher W, Friedman A (2020) Structural, Molecular, and Functional Alterations of the Blood-Brain Barrier during Epileptogenesis and Epilepsy: A Cause, Consequence, or Both? International journal of molecular sciences 21:591. https://doi.org/10.3390/ijms21020591

9. Aronica E, Bauer S, Bozzi Y, Caleo M, Dingledine R, Gorter JA, Henshall DC, Kaufer D, Koh S, Lцscher W, Louboutin JP, Mishto M, Norwood BA, Palma E, Poulter MO, Terrone G, Vezzani A, Kaminski RM (2017) Neuroinflammatory targets and treatments for epilepsy validated in experimental models. Epilepsia 58:2738. https://doi.org/10.1111/epi.13783

10. Sokolova TV, Zabrodskaya YM, Paramonova NM, Dobrogorskaya LN, Kuralbaev AK, Kasumov VR, Sitovskaya DA (2017) Apoptosis of brain cells in epileptic focus in pharmacoresistant temporal lobe epilepsy. Translational Medicine 4:22-33. (In Russ). https:// doi.org/10.18705/2311-4495-2017-4-6-22-33

11. Fuller OK, Whitham M, Mathivanan S, Febbraio MA (2020) The Protective Effect of Exercise in Neurodegenerative Diseases: The Potential Role of Extracellular Vesicles. Cells 9:2182. https://doi.org/10.3390/cells9102182

12. Yamanaka G, Morichi S., Takamatsu T, Wata- nabe Y, Suzuki S, Ishida Y, Oana S, Yamazaki T, Takata F, Kawashima H (2021) Links between Immune Cells from the Periphery and the Brain in the Pathogenesis of Epilepsy: A Narrative Review. International journal of molecular sciences 22:4395. https://doi.org/10.3390/ijms22094395

13. Sah E, Krishnamurthy S, Ahmidouch MY, Gillispie GJ, Milligan C, Orr ME (2021) The Cellular Senescence Stress Response in Post-Mitotic Brain Cells: Cell Survival at the Expense of Tissue Degeneration. Life 11:229. https://doi.org/ 10.3390/life 11030229

14. Spanoghe J, Larsen LE, Craey E, Manzella S, Van Dycke A, Boon P, Raedt R (2020) The Signaling Pathways Involved in the Anticonvulsive Effects of the Adenosine A1 Receptor. International journal of molecular sciences 22:320. https://doi.org/ 10.3390/ijms22010320

15. Bacher S, Meier-Soelch J, Kracht M, Schmitz ML (2021) Regulation of Transcription Factor NF- $\mathrm{KB}$ in Its Natural Habitat: The Nucleus. Cells. 10:753. https://doi.org/10.3390/ cells10040753

16. Jung YJ, Tweedie D, Scerba MT, Kim DS, Palmas MF, Pisanu A, Carta AR, Greig NH (2021) Repurposing Immunomodulatory Imide Drugs (IMiDs) in Neuropsychiatric and Neurodegenerative Disorders. Front Neurosci. 15:656921. https://doi.org/10.3389/fnins.2021.656921

17. Li G, Yamasaki R, Fang M, Masaki K, Ochi H, Matsushita T, Kira JI (2018) Novel disease-modifying anti-rheumatic drug iguratimod suppresses chronic experimental autoimmune encephalomyelitis by down-regulating activation of macrophages/microglia through an NF- $\kappa \mathrm{B}$ pathway. Sci Rep. 8:1933. https://doi.org/10.1038/s41598018-20390-5

18. Mao X-Y, Zhou H-H, Jin W-L (2019) RedoxRelated Neuronal Death and Crosstalk as Drug Targets: Focus on Epilepsy. Frontiers in Neuroscience 13:512. https://doi.org/10.3389/ fnins.2019.00512

19. Benusa SD, Lafrenaye AD (2020) Microglial process convergence on axonal segments in health and disease. Neuroimmunol Neuroinflamm. 7:2339. https://doi.org/10.20517/2347-8659.2019.28

20. Rana A, Musto AE (2018) The role of inflammation in the development of epilepsy. Journal of Neuroinflammation 15:144. https://doi.org/ 10.1186/s12974-018-1192-7

21. Mancini A, Ghiglieri V, Parnetti L, Calabresi P, Di Filippo M (2021) Neuro-Immune Cross-Talk in the Striatum: From Basal Ganglia Physiology to Circuit Dysfunction. Front Immunology 
$12: 644294$.

fimmu.2021.644294

https://doi.org/10.3389/

22. Rocha NKR, Themoteo R, Brentani H, Forlenza OV, De Paula VJR (2020) NeuronalGlial Interaction in a Triple-Transgenic Mouse Model of Alzheimer's Disease: Gene Ontology and Lithium Pathways. Front Neurosci 14:579984. https://doi.org/10.3389/fnins.2020.579984

23. Zheng P, Chen Q, Tian X, Qian N, Chai P, Liu B, Hu J, Blackstone C, Zhu D, Teng J, Chen J (2018) DNA damage triggers tubular endoplasmic reticulum extension to promote apoptosis by facilitating ER-mitochondria signaling. Cell Research 28:833-854. https://doi.org/10.1038/s41422018-0065-z

24. Pérez-Figueroa E, Álvarez-Carrasco P, Ortega E, Maldonado-Bernal C (2021) Neutrophils: Many Ways to Die. Front Immunology 12:631821. https://doi.org/10.3389/fimmu.2021.631821

25. Yu H, Lin L, Zhang Z, Zhang H, Hu H (2020) Targeting NF- $\kappa B$ pathway for the therapy of diseases: mechanism and clinical study. Signal Transduct Target Therapy 5:209. https://doi.org/ 10.1038/s41392-020-00312-6

26. Liang J, Luan Y, Lu B, Zhang H, Luo YN, Ge P
(2014) Protection of ischemic postconditioning against neuronal apoptosis induced by transient focal ischemia is associated with attenuation of NF- $\kappa$ B/p65 activation. PLoS One 9:e96734. https://doi.org/10.1371/journal.pone.0096734

27. Aubrey BJ, Kelly GL, Janic A, Herold MJ, Strasser A (2017) How does p53 induce apoptosis and how does this relate to p53-mediated tumour suppression? Cell Death and Differentiation 25:104-113. https://doi.org/10.1038/ cdd.2017.169

28. Simabuco FM, Morale MG, Pavan ICB, Morelli AP, Silva FR, Tamura RE (2018) p53 and metabolism: from mechanism to therapeutics. Oncotarget 9:23780-23823. https://doi.org/ 10.18632/oncotarget. 25267

29. Sazhina TA, Sitovskaya DA, Zabrodskaya YM, Bazhanova ED (2020) Functional Imbalance of Glutamate- and GABAergic Neuronal Systems in the Pathogenesis of Focal Drug-Resistant Epilepsy in Humans. Bulletin of Experimental Biology and Medicine 168:519-522. https://doi.org/ 10.1007/s10517-020-04747-3

Translated by A. Polyanovsky 